\title{
Linear versus nonlinear reliability-based topology optimization with application to bridge structures
}

\author{
Ghais Kharmanda ${ }^{1}$, Hasan Mulki ${ }^{2}$, and Antypas $\operatorname{Imad}^{3 *}$ \\ ${ }^{1}$ INSA Rouen, St Etienne du Rouvray, France \\ ${ }^{2}$ College of Engineering and Technology, American University of the Middle East, Kuwait \\ ${ }^{3}$ Don State Technical University, Gagarin square 1, 344003 Rostov-on-Don, Russian Federation
}

\begin{abstract}
In general, two types of topology optimization models can be found in literature. The first type is called Deterministic Topology Optimization (DTO) leading to a single layout when considering a given design space. The second type is called Reliability-Based Topology Optimization (RBTO) leading to various solutions. In our previous work, two strategies based on the Inverse Optimum Safety Factors (IOSF) were established and applied simply to the normal (Gaussian or linear) distribution law. In this work, linear and nonlinear (normal and lognormal) distribution RBTO cases are compared. A bridge structure is considered a numerical application to perform this comparison where different layouts at the same level of reliability can be found. The numerical results show that regarding the distribution laws, certain output parameters and safety factor values are affected. This change can affect the resulting topology layouts as well as the output parameter such as the compliance. When rising the values of the reliability index, the values of the compliance become larger and the volume values become lesser for the lognormal distribution when comparing to the normal one.
\end{abstract}

\section{Introduction}

Topology optimization is performed at the conceptual design stage where the objective is to obtain the best distribution of material density. This layout is essential for all following analysis and optimization measures. The classical Deterministic Topology Optimization (DTO) leads to a single layout, while the Reliability-Based Topology Optimization (RBTO) model leads to several solutions with special advantages. The different RBTO developments can be regrouped in two points of view:

The first point of view is 'topology optimization', the original RBTO form have been established with aim of supplying the designer with several reliability-based topology layouts, conversely in the classical topology optimization, the designer gets only a single deterministic one [1]. It has been demonstrated that the importance of the RBTO model

\footnotetext{
* Corresponding author: imad.antypas@mail.ru
} 
yields structures that should be more reliable than those provided by deterministic topology optimization (for the same weight, several applications can be found in [1]. In the RBTO form, reliability constraints have been integrated into DTO forms. The primary sensitivity analysis is utilized to choose the random variables which significantly affect the objective function. A Gradient-Based Method (GBM) was utilized to find the target value of reliability index, where the limit state function is assumed to be a linear combination of the random variables. Several works have been performed considering the same point of view $[2,3]$. Newly, [4] developed a hybrid method of RBTO with the aim of handling epistemic and aleatory uncertainties. This method consists of an efficient single optimization loop method based on Karush-Kuhn-Tucker optimality condition.

The second point of view is 'reliability analysis' where the classical topology optimization is to find the stiffest layout subject to a volume constraint. It was assumed in these studies that the feasibility of volume constraint is not critical in this structural study $[5,6]$.

Considering both points of view ('topology optimization' and 'reliability analysis'), the developments from a point of view 'reliability analysis' are essentially computationally expensive since additional required system analysis associate with RBDO. This way a large number of design variables are associated with topology optimization procedures. Consequently, the point of view 'topology optimization' seems to be attractive for topology designers since it supplies with several reliability-based configurations in function of reliability index changes. It leads to several configurations, but the developments from a point of view 'reliability analysis' provides same configurations with different densities that doesn't represent importance sense for the following optimization stages. Newly, two different approaches based on the IOSF (Inverse Optimum Safety Factor) are elaborated and applied to linear distribution law [7-9]. In this paper, an extension of these approaches is carried out considering nonlinear distribution laws such as lognormal distribution law.

\section{Materials and methods}

\subsection{Deterministic topology optimization}

Two ways are considered here to classify topology optimization problems [10-13]. The first way is called Objective-Based Approach (OBA), where we seek to minimize the structural compliance taking into account an allowable ratio decrease of volume ${ }_{f}^{V_{f}^{T}}$. The problem is mathematically formulated as:

$$
\begin{aligned}
& \min : C(\mathbf{x}) \\
& \text { s.t. }: \frac{V(\mathbf{x})}{V^{0}} \leq V_{f}^{T r}
\end{aligned}
$$

where $C(\mathbf{x})$ is the structural compliance taking into account the densities of the used material in each element as optimization variables. Their values vary in the interval $[0,1]$. $V^{0}$ and $V(\mathbf{x})$ are the values of the initial- and current structural volumes. Conversely, the first one is called Performance-Based Approach (PBA) where the objective is to minimize the structural volume taking into account an allowable ratio increase of compliance ${ }^{T_{f}^{T}}$. The problem is mathematically formulated as:

$$
\begin{aligned}
& \min : V(\mathbf{x}) \\
& \text { s.t.: } \frac{C(\mathbf{x})}{C^{0}} \leq 1+C_{f}^{T r}
\end{aligned}
$$


where $^{V(\mathbf{x})}$ is the structural volume taking into account the material densities in each element as optimization variables and vary in the interval $[0,1] . C^{0}$ and $C(\mathbf{x})$ represent the values of the initial- and current structural compliances.

\subsection{Reliability index}

A reliability index $\beta$ is considered here to provide several layouts by performing the transformation between the physical and normalized spaces (Fig. 1). The evaluation of the reliability index is generally carried out by the following optimization procedure $[14,15]$ :

$$
\begin{aligned}
& \beta=\min d(\mathbf{u}) \\
& \text { s.t.: } H(\mathbf{u})=0
\end{aligned}
$$

where $^{d(\mathbf{u})}$ is the distance the normalized space between the limit state curve and the origin. This distance is written as:

$$
d(\mathbf{u})=\sqrt{\sum_{i=1}^{n} u_{i}^{2}}
$$

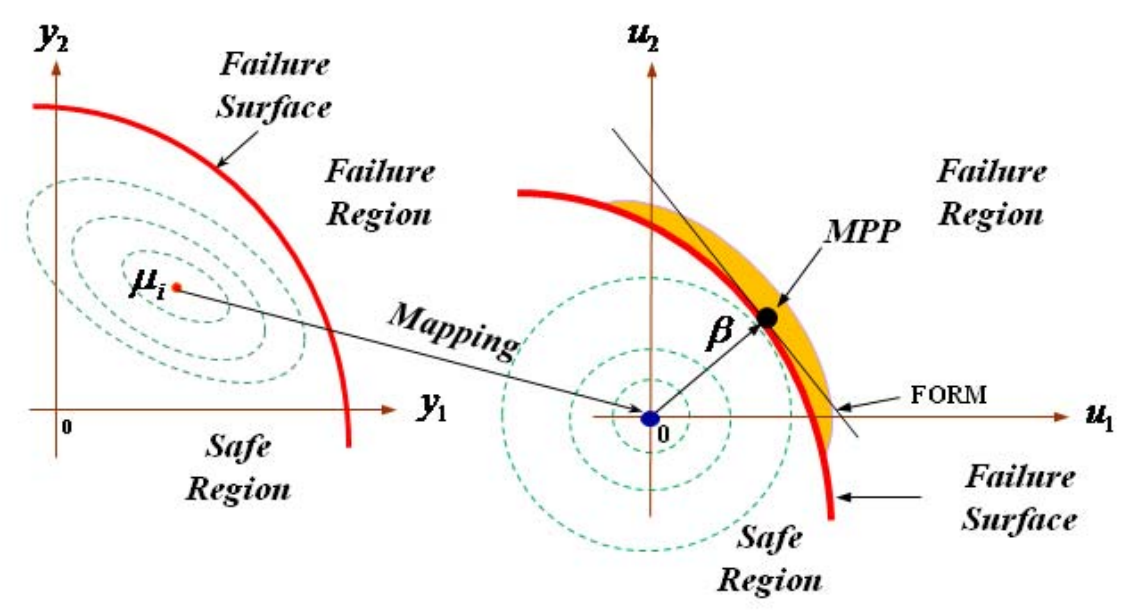

Fig. 1. Transformation image between the physical space and the normalized one.

\subsection{Reliability-based topology optimization}

\subsubsection{Objective-Based IOSF Approaches (OBA-IOSF)}

Considering the Objective-Based Approaches, the problem of RBTO model is to minimize the structural compliance taking into account an allowable ratio decrease of volume ${ }^{V_{f}^{T_{r}}}$ and the reliability constraint. The problem is mathematically formulated as:

$$
\begin{aligned}
& \min : C(\mathbf{x}) \\
& \text { s.t. }: \beta \geq \beta_{t} \\
& \text { and }: \frac{V(\mathbf{x})}{V^{0}} \leq V_{f}^{T r}
\end{aligned}
$$


where $^{\beta_{t}}$ is the target value of reliability index. For this kind of problems, the failure criterion is associated to the compliance. But, in this case, the compliance is considered to be the objective function. Hence, the sensitivity is estimated for the objective function. This way the optimum value of the normalized vector can be written by:

$$
u_{i}^{O p t}= \pm \sqrt{\frac{\left|\frac{\partial F}{\partial y_{i}}\right|}{\sum_{j=1}^{n}\left|\frac{\partial F}{\partial y_{j}}\right|}}
$$

taking into account the sign of the objective function derivative with respect to random variables ${ }^{y_{i}}$, we have for the objective-based iosf approach:

$$
\frac{\partial F}{\partial y_{i}}>0 \Leftrightarrow u_{i}^{O p t}>0 \quad \frac{\partial F}{\partial y_{i}}<0 \Leftrightarrow u_{i}^{O p t}<0
$$

\section{Results and discussion}

Considering the Performance-Based Approaches, the RBTO problem consists of minimizing the structural volume taking into account the compliance constraint and the reliability one. The problem is mathematically formulated as:

$$
\begin{aligned}
& \min : V(\mathbf{x}) \\
& \text { s.t. } \beta \geq \beta_{t} \\
& \text { and }: \frac{C(\mathbf{x})}{C^{0}} \leq 1+C_{f}^{t}
\end{aligned}
$$

The optimum value of the normalized vector can be expressed by:

$$
u_{i}^{O p t}= \pm \beta_{t} \sqrt{\frac{\left|\frac{\partial G}{\partial y_{i}}\right|}{\sum_{j=1}^{n}\left|\frac{\partial G}{\partial y_{j}}\right|}}
$$

taking into account the sign of the objective function derivative with respect to random variables $y_{i}$, we have for the performance-based iosf approach:

$$
\frac{\partial G}{\partial y_{i}}>0 \Leftrightarrow u_{i}^{O p t}>0 \quad \frac{\partial G}{\partial y_{i}}<0 \Leftrightarrow u_{i}^{O p t}<0
$$

\subsection{Probabilistic distribution laws}

To apply these approaches, the types of the distribution laws should be selected. In this work, the normal and lognormal distribution are used as linear and nonlinear distributions, respectively. The optimum safety factor for normal (Gauss) distribution, can be expressed by [1]: 


$$
S_{f_{i}}=1+\lambda_{i} \cdot u_{i}^{*}
$$

On the other hand, the optimum safety factor formulation for the lognormal distribution can be presented as follows [1]:

$$
S_{f_{i}}=\frac{1}{\sqrt{1+\lambda_{i}^{2}}} \exp \left(\sqrt{\ln \left(1+\lambda_{i}^{2}\right)} \cdot u_{i}^{*}\right), \quad i=1, \ldots, n
$$

where $\lambda_{i}=\sigma_{i} / \mu_{i}$ represents the relationship between the mean value ${ }^{\mu_{i}}$ of the random variable ${ }^{y_{i}}$ and its standard-deviation ${ }^{\sigma_{i}}$.

Here, the failure point $P_{y}^{*}$ is represented by the starting point and an RBTO layout $P_{x}^{*}$ is next obtained. This point $P_{x}^{*}$ must meet a target reliability index ${ }^{\beta_{t}}$. The DTO procedure is utilized to reach the failure point $P_{y}^{*}$.

\section{Results}

A simple 2D bridge model is illustrated in Fig. 2. The topology optimization is performed considering two ways: In the first way (OBA), the optimization problem is to minimize the compliance ${ }^{C(\mathbf{x})}$ taking into account the constraint of the volume decrease ratio ${ }_{f}^{V_{r}^{\pi}}$ (Eqs 1 and 5). However, in the second way (PBA), the optimization problem is to minimize the volume ${ }^{V(\mathbf{x})}$ taking into account the constraint of the compliance increase ratio ${ }_{f}^{T_{r}}$ (Eqs 2 and 8).

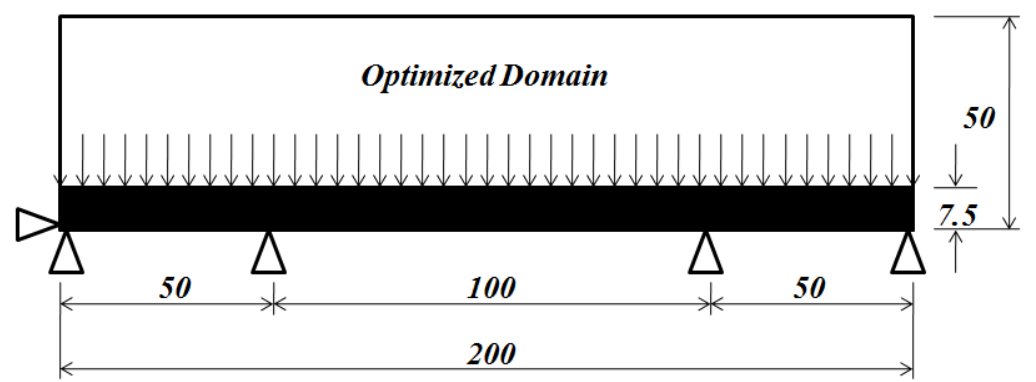

Fig. 2. Initial configuration of the studied $2 \mathrm{D}$ bridge structure.

The original domain is modeled by a rectangle $(200 \times 50 \mathrm{~m})$. As shown in Fig. 2, This area contains two subareas: the dimensions of the lower rectangle being the non-optimized domain, are: $200 \times 7.5 \mathrm{~m}$, but the upper one is assumed to be optimized where its dimensions are: $200 \times 42.5 \mathrm{~m}$. The material of this bridge is steel. It is linear elastic isotropic where the Young's modulus is: $E=200000(\mathrm{MPa})$ and the Poisson's ratio is: $v=0.3$. The bridge is submitted to a distributed force is: $\mathrm{F}=1 \times 108(\mathrm{~N} / \mathrm{m})$. The fixation points are shown in Fig.2. Two categories of solutions are obtained according to the developed approach. Each category contains two sub-categories according to the distribution laws (normal and lognormal).The topology optimization procedures are carried out using ANSYS software. The Optimality Criteria (OC) is used for the first category of solutions (DTO by OBA and RBTO by OBA-IOSF) and the Sequential Convex Programming (SCP) is used for the second category of solutions (DTO by PBA and RBTO by PBA-IOSF). 


\subsection{First category of solutions}

The starting configuration is considered to be the failure point where the optimization problem is to minimize the structural compliance subject to the volume constraint for the DTO (Eq. 1) and also the reliability constraints for the RBTO (Eq. 5). Fig. 3a represents the resulting DTO layout using OBA [9]. Figs $3 b$ and c illustrate the resulting RBTO layouts using OBA-IOSF at $\beta_{t}=3$, for normal and lognormal distribution, respectively. Figs $3 \mathrm{~d}$ and e illustrate the resulting RBTO layouts using OBA-IOSF at $\beta_{t}=3.8$, for normal and lognormal distribution, respectively. Figs $3 \mathrm{f}$ and $\mathrm{g}$ illustrate the resulting RBTO layouts using OBA-IOSF at $\beta_{t}=4.25$, for normal and lognormal distribution, respectively. For OBA-IOSF, Table 1 shows the resulting values of the safety factors, compliance and volume at the optimal configuration for normal and lognormal distribution considering three values of reliability index. $E, v, F$ and $V_{f}^{T_{r}}$ are considered as random variables. The $\lambda_{i}=0.05 \mathrm{~s}$ is considered the proportional coefficient between the mean and the standard deviation.

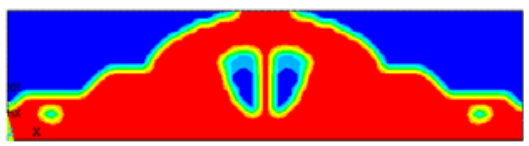

$a$

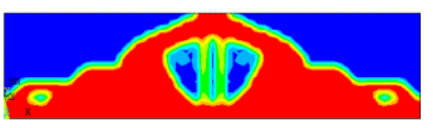

b

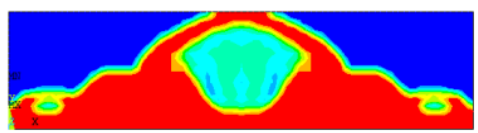

$\boldsymbol{d}$

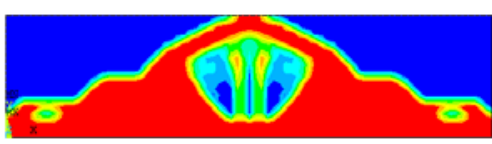

$f$

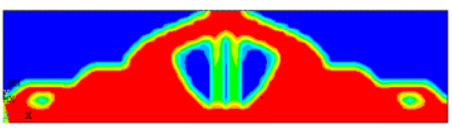

$c$

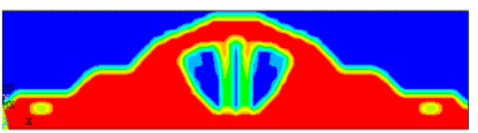

$\boldsymbol{e}$

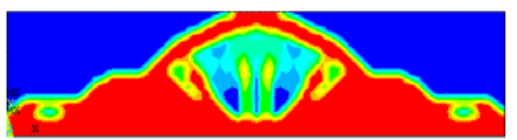

$g$

Fig. 3. a) DTO layout using OBA [9]. b) and c) RBTO layouts using OBA-IOSF at $\beta_{t}=3$, for normal and lognormal distribution, respectively. d) and e) RBTO layouts using OBA-IOSF at $\beta_{t}=3.8$ , for normal and lognormal distribution, respectively. f) and g) RBTO layouts using OBA-IOSF at $\beta_{t}=4.25$, for normal and lognormal distribution, respectively. 
Table 1 shows the values of the safety factors, the compliance and the volume at the reliability-based optimal configuration using OBA-IOSF and considering the three selected target values of reliability index for the normal and lognormal distribution laws.

Table 1. Different RBTO result using OBA-IOSF.

\begin{tabular}{|c|c|c|c|c|c|c|c|}
\hline \multirow{3}{*}{ Parameters } & \multirow{3}{*}{ DTO } & \multicolumn{6}{|c|}{ RBTO using $O B A-I O S F$} \\
\hline & & \multicolumn{2}{|c|}{$\beta_{t}=3$} & \multicolumn{2}{|c|}{$\beta_{t}=3.8$} & \multicolumn{2}{|c|}{$\beta_{t}=4.25$} \\
\hline & & Normal & Lognormal & Normal & Lognormal & Normal & Lognormal \\
\hline$S_{f}^{E}$ & & 0.997209 & 1.001542 & 0.996465 & 1.002287 & 0.996046 & 1.002707 \\
\hline$S_{f}^{F}$ & & 1.000177 & 0.998929 & 1.000224 & 0.998976 & 1.00025 & 0.999002 \\
\hline$S_{f}^{v}$ & & 1 & 0.998752 & 1 & 0.998752 & 1 & 0.998752 \\
\hline$S_{f}^{V_{f}^{T r}}$ & & 1.149974 & 1.160246 & 1.189967 & 1.207558 & 1.212463 & 1.235014 \\
\hline$C^{*}$ & $\begin{array}{c}4.61 \\
\times \\
10^{14} \\
\end{array}$ & $\begin{array}{c}5.14^{\times} \\
10^{14}\end{array}$ & $5.15 \times 10^{14}$ & $\begin{array}{c}5.48^{\times} \\
10^{14}\end{array}$ & $5.30 \times 10^{14}$ & $\begin{array}{c}5.45^{\times} \\
10^{14}\end{array}$ & $5.53 \times 10^{14}$ \\
\hline$V(\mathbf{x})$ & 4250 & 3612 & 3569 & 3442 & 3368 & 3347 & 3251 \\
\hline
\end{tabular}

\subsection{Second category of solutions}

The starting configuration is considered to be the failure point where the optimization problem is to minimize the volume subject to the compliance constraint for the DTO (Eq. 2) and also the reliability constraints for the RBTO (Eq. 9). Fig. 4a represents the resulting DTO layout using PBA [9]. Figs 4b and c illustrate the resulting RBTO layouts using PBAIOSF at $\beta_{t}=3$, for normal and lognormal distribution, respectively. Figs $4 \mathrm{~d}$ and e illustrate the resulting RBTO layouts using PBA-IOSF at $\beta_{t}=3.8$, for normal and lognormal distribution, respectively. Figs $4 \mathrm{f}$ and g illustrate the resulting RBTO layouts using PBAIOSF at $\beta_{t}=4.25$, for normal and lognormal distribution, respectively. For PBA-IOSF, Table 2 illustrates the resulting values of the safety factors, compliance and volume at the optimal configuration for normal and lognormal distribution considering three values of reliability index. $E, v, F$ and $C_{f}^{T_{r}}$ are considered as random variables. The $\lambda_{i}=0.05 \mathrm{~s}$ is considered the proportional coefficient between the mean and the standard deviation. 


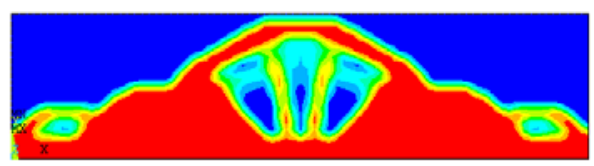

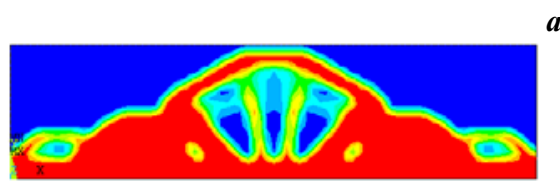

b

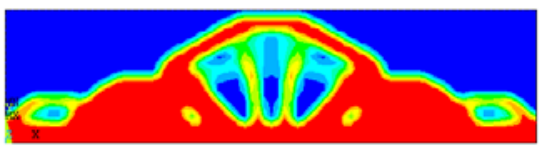

$d$

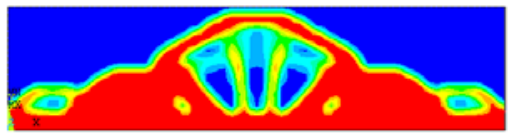

$f$

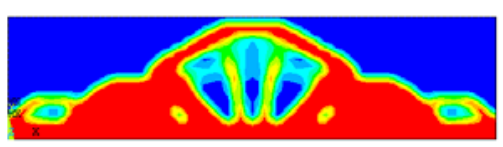

$c$

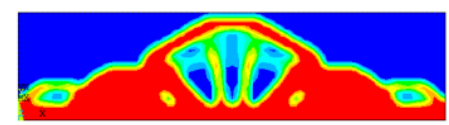

$\boldsymbol{e}$

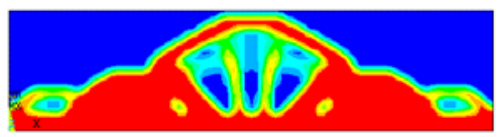

$g$

Fig. 3. a) DTO layout using PBA [9]. b) and c) RBTO layouts using PBA-IOSF at $\beta_{t}=3$, for normal and lognormal distribution, respectively. d) and e) RBTO layouts using PBA-IOSF at $\beta_{t}=3.8$, for normal and lognormal distribution, respectively. f) and g) RBTO layouts using PBA-IOSF at $\beta_{t}=4.25$ , for normal and lognormal distribution, respectively.

Table 2 shows the values of the safety factors, the compliance and the volume at the reliability-based optimal configuration using PBA-IOSF and considering the three selected target values of reliability index for the normal and lognormal distribution laws.

Table 2. Different input and output parameters for the second category of solutions.

\begin{tabular}{|c|c|c|c|c|c|c|c|}
\hline \multirow{3}{*}{ Parameters } & \multirow{3}{*}{ DTO } & \multicolumn{6}{|c|}{ RBTO using PBA-IOSF } \\
\hline & & \multicolumn{2}{|c|}{$\beta_{t}=3$} & \multicolumn{2}{|c|}{$\beta_{t}=3.8$} & \multicolumn{2}{|c|}{$\beta_{t}=4.25$} \\
\hline & & Normal & Lognormal & Normal & Lognormal & Normal & Lognormal \\
\hline$S_{f}^{E}$ & \multirow{4}{*}{--- } & 0.998095 & 1.000656 & 0.997587 & 1.001164 & 0.997301 & 1.00145 \\
\hline$S_{f}^{F}$ & & 1.000121 & 0.998873 & 1.000153 & 0.998905 & 1.000171 & 0.998923 \\
\hline$S_{f}^{v}$ & & 1.13288 & 1.140593 & 1.168315 & 1.181708 & 1.188246 & 1.205483 \\
\hline$S_{f}^{C_{f}^{T_{r}}}$ & & 1.069565 & 1.070658 & 1.088116 & 1.090692 & 1.09855 & 1.102125 \\
\hline$C^{*}$ & $\begin{array}{c}5.75^{\times} \\
10^{14} \\
\end{array}$ & $\begin{array}{c}6.01^{\times} \\
10^{14} \\
\end{array}$ & $5.98 \times 10^{14}$ & $\begin{array}{c}6.08^{\times} \\
10^{14} \\
\end{array}$ & $6.04 \times 10^{14}$ & $\begin{array}{c}6.13^{\times} \\
10^{14} \\
\end{array}$ & $6.09 \times 10^{14}$ \\
\hline$V(\mathbf{x})$ & 3148.39 & 3051 & 3050 & 3026 & 3024 & 3008 & 3006 \\
\hline
\end{tabular}

Fig. 4 illustrates the evolution of the output parameters (compliance and volume) in function of the reliability index changes. In this Figure, some abbreviations are taken into account. These abbreviations are classified according to the output parameters ( $\mathrm{C}$ refers to the compliance and $\mathrm{V}$ refers to the volume), the used approaches (OI refers to the OBAIOSF and PI refers to PBA-IOSF) and the distribution laws ( $\mathrm{N}$ refers to Normal distribution and $\mathrm{L}$ refers to the Lognormal one). 


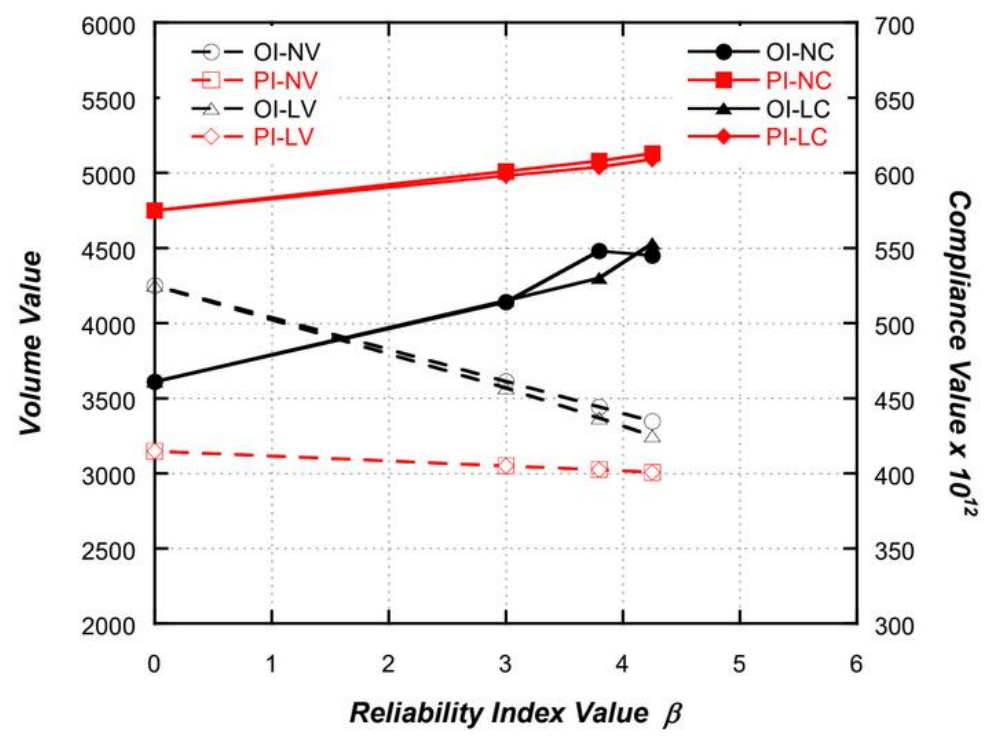

Fig. 4. Modeling of the change of the compliance and the volume in function of reliability index values.

\section{Discussion and conclusions}

Two developed RBTO approaches based on IOSF are extended to nonlinear distribution case. This leads to two sub-categories of solutions for each category.

When considering the RBTO by OBA-IOSF, the optimization problem seeks to minimize the compliance subject to the volume constraint. A sensitivity study of the compliance being the objective function, is carried out in [9], considering four random variables. It is found that the biggest value corresponds the volume fraction, however there is no role for the Poisson's ratio. It supplies the designer with the first RBTO category of solutions in function of the change of the reliability index. There is a decrease of the volume value $^{V(\mathbf{x})}$, when the reliability index values increase (Table 1).

However, when considering the RBTO by PBA-IOSF, the optimization problem seeks to minimize the volume subject to the compliance constraint. A sensitivity study of the compliance being the performance function, is carried out in [9], considering four random variables. It is found that the biggest value corresponds the Poisson's ratio, however the smallest value corresponds to the applied force. When increasing the values of reliability index, the resulting Poisson's ratio exceeds its limit $(\varepsilon>0.4)$. Therefore, the standarddeviation/mean rate is considered $5 \%$ instead of $10 \%$. It supplies the designer with the second RBTO category of solutions in function of the change of the reliability index. There is a small decrease of the volume value $V(\mathbf{x})$, when the reliability index values increase (Table 2).

In addition, when considering the safety factor values, there are different effects for certain parameters such as Young's modulus $E$ and the applied force $F$ as shown in Tables 1 and 2. For the same parameter, the normal distribution leads to a safety factor bigger than one $\left({ }^{S_{f}}>1\right)$, while the lognormal leads a value smaller than one (see Equations 13 and 14). This difference do not largely affect the output parameter (compliance and 
volume). According to Fig. 4, there is no big difference regarding the distribution laws. It is almost similar. This is because the sensitivity of the compliance with respect to Young's modulus $E$ and the applied force $F$ is very small as shown before in [9].

The RBTO approaches provide two categories of solution. Each category leads to several sub-categories when considering several distribution laws. The two main categories are dependent on the used method, however the sub-categories are dependent on the used distribution law. These sub-categories can be extended to others distribution laws.

\section{References}

1. G. Kharmanda, A. El-Hami, Biomechanics: Optimization, Uncertainties and Reliability, ISTE \& Wiley, 254 (2017)

2. J. Patel, S.K. Choi, Structural and Multidisciplinary Optimization, 45(4), 529-543, (2012)

3. L. Wang, D. Liu, Y. Yang, X. Wang, Z. Qiu, Computer Methods in Applied Mechanics and Engineering, 326, 573-595 (2017)

4. Z. Meng, Y. Pang, Y. Pu, X. Wang, Computer Methods in Applied Mechanics and Engineering, 363, 112886, (2020)

5. Y.S. Eom, K.S. Yoo, J.Y. Park, S.Y. Han, Structural and Multidisciplinary Optimization, 43 (2), 287-295 (2011)

6. M. Jalalpour, M. Tootkaboni, Journal of Structural and Multidisciplinary Optimization, 53, 759-772 (2016)

7. G. Kharmanda, I. Antypas, A. Dyachenko, Journal of Engineering Technologies and Systems, 29(1), 8-19 (2019)

8. G. Kharmanda, I. Antypas, A. Dyachenko, Journal of Engineering Technologies and Systems, 29 (3), 332-344, (2019)

9. G. Kharmanda, I. Antypas, A. Dyachenko, Journal of Engineering Technologies and Systems, 30(3), 498-511 (2020)

10. O. Sigmund, K. Maute, Structural and Multidisciplinary Optimization, 48, 1031-1055, (2013)

11. N. Jain, S. Bankoti, R. Saxena, Journal for Research in Emerging Science and Technology, 2 (2), 41-47, (2015)

12. W. Zhang, J. Zhu, T. Gao, Topology Optimization in Engineering Structure Design, ISTE \& Elsevier, 294, (2016)

13. N. Strömberg, Engineering Optimization, 52 (7), 1136-1148, (2020)

14. P. Thoft-Cristensen, M.J. Springer, Science \& Business Media, 268, (2012)

15. Y-G. Zhao-Hui Lu Z-H. Structural Reliability: Approaches from Perspectives of Statistical Moments, Wiley-Blackwell (2021) 